\title{
Notch1-dependent regulation of p27 determines cell fate in colorectal cancer
}

\author{
NEVYANA R. HRISTOVA ${ }^{1}$, KATRIN E. TAGSCHERER ${ }^{1}$, ANNE FASSL ${ }^{1}$, \\ JUERGEN KOPITZ ${ }^{2}$ and WILFRIED ROTH ${ }^{1,2}$ \\ ${ }^{1}$ Molecular Tumor Pathology, German Cancer Research Center (DKFZ); ${ }^{2}$ Institute of Pathology, \\ University of Heidelberg, D-69120 Heidelberg, Germany
}

Received July 17, 2013; Accepted September 2, 2013

DOI: $10.3892 /$ ijo.2013.2140

\begin{abstract}
Enhanced Notch signaling contributes to uncontrolled cell growth and cell death resistance in cancer. Here, we demonstrate that in colorectal carcinoma cells the Notch1-dependent activation of cell cycle and proliferation is mediated by repression of the cyclin-dependent kinase inhibitor (CDKI) p27. The half-life of p27 significantly increased after siRNA-mediated knockdown of Notch1. Notch1 depletion altered the transcription of SKP2, KPC1 and KPC2, which are E3-ubiquitin ligase subunits targeting p27 for proteasomal degradation in the nucleus and the cytoplasm, respectively. As a consequence, the levels of p27 in both cellular fractions were elevated upon Notch1 knockdown. Importantly, the downregulation of Notch1 significantly sensitized colorectal cancer cells to chemotherapy and ionizing radiation. Our findings support an important role of p27 in Notch1-dependent oncogenic signaling and suggest that Notch1 is a promising target for an experimental therapy of colorectal carcinoma.
\end{abstract}

\section{Introduction}

Notch signaling is highly conserved throughout the animal kingdom (1). It plays a crucial role in embryonic development and is implicated in the regulation of cellular proliferation, differentiation, apoptosis and angiogenesis in adult organisms $(2,3)$. This pathway is essential for normal intestinal homeostasis by controlling stem cell fate decisions (4). All Notch receptors and ligands as well as several target genes (Hes1, 5, 6, 7 and Math1) are expressed at various stages of development and differentiation in mouse intestinal crypts, the niche of intestine stem/progenitor cells (5). Inhibition of the Notch signaling pathway via Hes1-depletion is accompanied by

Correspondence to: Dr Wilfried Roth, Molecular Tumor Pathology, German Cancer Research Center (DKFZ), Im Neuenheimer Feld 280 , D-69120 Heidelberg, Germany

E-mail: w.roth@dkfz.de

Key words: Notch1, p27, SKP2, KPC1, KPC2, cell death, cell proliferation, colorectal cancer an increase of the secretory cells at the expense of absorptive enterocytes (6). Additionally, this pathway is involved in cell cycle progression of crypt progenitor cells (7). Because of its crucial role in cell fate decision, it is not surprising that Notch signaling has been implicated in colorectal cancer (CRC) development. Overexpression of Notch receptors, ligands and downstream targets is observed in most CRC tissues compared to normal colonic tissues which is associated with activation of the pathway (8).

In mammals there are four Notch receptors (Notch 1, 2, 3,4 ) and five Notch ligands (Jagged 1,2, Delta-like 1, 3, 4), all of which are transmembrane proteins. Two proteolytic cleavage steps are required for activation of the receptor upon its interaction with a ligand (9). The first (S2) cleavage detaches the Notch extracellular domain and is catalyzed by ADAM-family metalloproteases, ADAM10 or ADAM17/TACE (10). The second (S3) cleavage is executed by the $\gamma$-secretase complex, leading to the release of the Notch intracellular domain (NICD) (11). The latter translocates to the nucleus where it interacts with the transcriptional regulator RBPJ and its co-activator Mastermind (Mam). This triggers transcription of Notch target genes, such as those belonging to the Hes and Hey families (12).

Several downstream effectors mediating the effects of Notch on cellular proliferation and differentiation have been described. One of them, $\mathrm{p} 27^{\mathrm{Kip} 1}$, belongs to the CIP/KIP family of cyclin-dependent kinase (CDK) inhibitors (CDKIs) which also includes $\mathrm{p} 21^{\mathrm{Cip} 1 / \mathrm{WAF} 1}$ and $\mathrm{p} 57^{\mathrm{Kip} 2}$. It accumulates during quiescence (G0) and needs to be degraded before a cell can enter the cell cycle. p27 is mainly regulated by post-transcriptional mechanisms (13), including diverse phosphorylation events and proteasomal degradation. To enable the cells to enter $\mathrm{S}$ phase, nuclear p27 levels have to decrease which is realized by SKP1-cullin-F-box protein $\left(\mathrm{SCF}^{\mathrm{SKP} 2}\right)$ (14). The prerequisite for this ubiquitination is a phosphorylation of $\mathrm{p} 27$ at threonine 187 (T187) by the CDK2-cyclin E/A complex. Another ubiquitin ligase, called Kip1 ubiquitination-promoting complex (KPC), accomplishes the degradation of p27 in the cytoplasm during early G1 phase (15). This ubiquitination is also phosphorylation-dependent since KIS and DYRK1 kinases can phosphorylate nuclear p27 at serine 10 (S10), thereby allowing p27 binding to CRM1 (also known as exportin 1) via its nuclear export signal (NES) (16). This leads to the export of p27 to the cytoplasm where it becomes a target for KPC-mediated 
proteasomal degradation. However, the precise mechanisms governing the Notch-dependent regulation of p27 are far from clear. Given the involvement of Notch in intestinal tissue homeostasis, the Notch-p27 axis could play a pivotal role in the development and progression of CRC.

$\mathrm{CRC}$ is on third and second place in terms of incidence and mortality amongst all malignancies, and the established therapies against advanced stages of the disease have only limited efficacy. Because Notch signaling is essential for stem cell fate decision, therapies targeting this pathway might improve CRC treatment. Due to the crucial role of Notch signaling in colon tissue, understanding the mechanisms of proliferation activation and apoptosis inhibition by this pathway is of major importance in order to develop novel promising antitumor therapies for patients suffering from CRC. In this study, we characterized the regulation of p27 by Notch1 and the effects of Notch1 signaling on cell growth. Moreover, we investigated the potential beneficial interference with Notch1 signaling for increasing the efficiency of chemo- and radiotherapy for treatment of CRC.

\section{Materials and methods}

Materials. The following reagents were used: oxaliplatin (Sigma, Deisenhofen, Germany, O9512), 5-fluorouracil (AppliChem, Darmstadt, Germany, A7686,0005), cycloheximide (Calbiochem, San Diego, CA, USA, 239763). The primary antibodies used in the experiments were: anti-Notch1 (Santa Cruz, San Diego, CA, USA, sc-6014), anti-p27 (Santa Cruz, sc-1641), anti-GAPDH (Santa Cruz, sc-32233), anti- $\beta$-actin (Chemicon International, Temecula, CA, USA, MAB1501), anti-KPC1 (Santa Cruz, sc-101122), anti-KPC2 (Abgent, San Diego,CA, USA, AP5353b), anti-SKP2 (Cell Signaling, Danvers, MA, USA, 4313), anti-PARP (BD Biosciences, San Jose, CA, USA, 556362), anti-caspase-3 (Imgenex, San Diego, CA, USA, IMG-144A) and anti-Ki67 (Dako, Glostrup, Denmark, M7240).

Cell culture. Human colorectal cancer cell lines were purchased from ATCC (Rockville, MD, USA), expanded and frozen in aliquots within four weeks. For the experiments described here, the cells were thawed and cultured for no more than eight weeks. Cell lines were authenticated by SNP profiling (17) and tested regularly for contaminations by multiplex PCR performed in the core facility of the DKFZ (18). All cell lines except $\mathrm{Caco} 2$ were grown in RPMI medium (Invitrogen, Carlsbad, CA, USA, 21875-034) containing 10\% FCS (PAA, Piscataway, NJ, USA, A15-151) and $1 \%$ penicillin/streptomycin (Invitrogen, 15140-122). Caco2 cells were cultured in MEM medium (Invitrogen, 31095-029) supplemented with 20\% FCS, $1 \%$ penicillin/streptomycin, 1\% MEM non-essential amino acids (PAA, M11-003), 1 mM sodium pyruvate (Sigma, S8636) and 1\% GlutaMAX (Invitrogen, 35050-038). All cell lines were cultured at $37^{\circ} \mathrm{C}$ in a $5 \% \mathrm{CO}_{2}$ atmosphere.

Transfections. Knockdown of endogenous proteins was achieved by transiently transfecting cell lines with short interfering RNA (siRNA) oligonucleotides using Lipofectamine 2000 (Invitrogen, 11668-019). siRNA oligonucleotides for Notch1 were obtained from Ambion [Austin, TX, USA, 144335 (si1), 108983 (si2)] and Dharmacon [Lafayette, CO, USA, D-007771-08 (si3)]; p27 siRNA (J-003472-07), KPC1 siRNA
[D-007041-01 (si1), D-007041-03 (si2)], and SKP2 siRNA [D-003324-07 (si1), D-003324-13 (si2)] were obtained from Dharmacon. Non-targeting siRNA pool was used as a control (Dharmacon, D-001810-10-20). The following siRNA concentrations were used for transfection: Notch1 siRNAs, $25 \mathrm{nM}$; KPC1 and SKP2 siRNAs, $20 \mathrm{nM}$; 27 siRNA, $10 \mathrm{nM}$.

Immunoblot analysis. Total cell lysates were prepared using lysis buffer [120 mM NaCl, $50 \mathrm{mM}$ Tris- $\mathrm{HCl}$ (pH 8.0), 5 mM EDTA, $0.5 \%$ Triton $\mathrm{X}-100]$ containing phenylmethylsulfonylfluoride (1 mM), proteinase inhibitors (Roche, Mannheim, Germany, $11697498001)$ and phosphatase inhibitors ( $25 \mathrm{mM} \mathrm{NaF}, 200 \mu \mathrm{M}$ $\mathrm{NaVO}_{3}, 10 \mathrm{mM} \mathrm{NaPPi}$ ). For cytoplasmic and nuclear fractions cells were harvested and processed with the Nuclear Extraction kit (Active Motif, Rixensart, Belgium, 40010) according to the manufacturer's protocol. Protein concentration was measured using the Bradford Assay (Bio-Rad, Munich, Germany, 500-0006). Soluble proteins (10-50 $\mu \mathrm{g}$ per lane) were separated on 10 or $12 \%$ SDS polyacrylamide gels and blotted on nitrocellulose membrane (Bio-Rad, 162-0115). Membranes were incubated with primary and secondary antibody (horseradish peroxidase-conjugated, Bio-Rad) which was visualized using an enhanced chemiluminescence detection system (GE Healthcare, Uppsala, Sweden, RPN2109).

Quantitative real-time PCR. Total RNA was isolated with the RNeasy Plus mini kit (Qiagen, Germantown, MD, USA, 74134). A total of $1 \mu \mathrm{g}$ RNA was reverse-transcribed to cDNA using SuperScript II (Invitrogen, 18064-014) and Random Hexamers (Applied Biosystems, Carlsbad, CA, USA, P14122). Quantitative real-time PCR was performed using SYBR-Green ${ }^{\circledR}$ PCR Master mix (Applied Biosystems, 4309155) and a 7300 Real-Time PCR system (Applied Biosystems). The following primers were utilized: Notch1 5'-GGGCCCTGAATTTCA CTGT-3' (forward), 5'-CGCAGAGGGTTGTATTGGTT-3' (reverse); p27 5'-AAAAATCCGAGGTGCTTGG-3' (forward), 5'-ACAGCCCGAAGTGAAAAGAA-3' (reverse); KPC1 5'-GTC CAAATGTTCTGGCAGGT-3' (forward), 5'-TGAACCGCATC TTTTCCTCT-3' (reverse); KPC2 5'-CATGTTGTAGGAGG GCAGGT-3' (forward), 5'-CCCAAGATGGCTGATGTCTC-3' (reverse); SKP2 5'-GAAGGGAGTCCCATGAAACA-3' (forward), 5'-CCAGGAACTGCTCTCAAACC-3' (reverse); 18S 5'-CATGGCCGTTCTTAGTTGGT-3' (forward), 5'-ATGC CAGAGTCTCGTTCGTT-3' (reverse).

Flow cytometry. Flow cytometry analysis was performed on BD Biosciences FACSCalibur flow cytometer using Cell Quest software. For measuring cell death, cells were incubated with trypsin, harvested, washed and stained with $50 \mu \mathrm{g} / \mathrm{ml}$ propidium iodide (Sigma, P4170) in Nicoletti buffer containing $50 \mu \mathrm{g} / \mathrm{ml}$ RNase (AppliChem, A3832,0050). For cell cycle distribution analysis, cells were washed, incubated with trypsin, harvested, washed, fixed in $75 \%$ ethanol for $1 \mathrm{~h}$ at $4^{\circ} \mathrm{C}$, washed and stained with $50 \mu \mathrm{g} / \mathrm{ml}$ propidium iodide in PBS containing $50 \mu \mathrm{g} / \mathrm{ml}$ RNase.

Immunohistochemistry. Cells were washed, incubated with trypsin, harvested, washed and fixed in formaldehyde for $15 \mathrm{~min}$ at $37^{\circ} \mathrm{C}$. After washing, the cells were dissolved in $100 \%$ ethanol to which $30 \%$ FCS in PBS was added (in propor- 
tion 5:1). Paraffin-embedded cells and tissue sections were dewaxed and rehydrated using xylene and a series of graded alcohols, followed by heat-induced antigen retrieval using a target retrieval solution (Dako, S2031) in a pressure cooker for $15 \mathrm{~min}$. The human tissue samples were provided by the Tumor Tissue Bank of the NCT Heidelberg after approval by the ethics committee of the University of Heidelberg. The collection of tissue samples comprised 44 primary colorectal adenocarcinomas with pT stadium pT3 and the following features: G2, $n=25 ; \mathrm{G} 3, \mathrm{n}=19 ; \mathrm{pN} 0, \mathrm{n}=25 ; \mathrm{pN} 1 / 2, \mathrm{n}=19$. Staining was done on an automated staining system (Techmate 500, Dako) with avidin-biotin-complex peroxidase technique using aminoethylcarbazole for visualization and haematoxylin for counterstaining. The sections were incubated with primary antibody overnight at $4^{\circ} \mathrm{C}$ [anti-Notch1 $(50 \mathrm{ng} / \mathrm{ml})$, anti-p27 $(5 \mu \mathrm{g} / \mathrm{ml})$ and anti-Ki67 (1:400)] and processed according to the manufacturer's instructions for the following kits: ChemMate Detection kit (Dako, K5003), ChemMate Buffer kit (Dako, K5006), Avidin/Biotin Blocking kit (Vector Laboratories, Burlingame, CA, USA, SP-2001). For the immunohistochemical semi-quantitative assessment of p27 expression, the staining intensity of immunoreactive tumor cells was determined based on the following scoring system: the intensity ranged from 0 , negative; 1 , low; 2 , medium to 3 , high. For statistical analysis, negative and low p27 expression was regarded as 'p27 low' and medium and high p27 expression was regarded as 'p27 high'. Proliferative activity of the tumors was assessed by counting Ki67-positive cells in the tumor tissue: 'Ki67 low' was attributed to tumors showing $<50 \% \mathrm{Ki} 67$-positive tumor cells, and 'Ki67 high' tumors showing $\geq 50 \%$ Ki67-positive tumor cells.

Clonogenicity assay. Cells were transfected with control, Notch1 and/or p27 siRNA. After 24 h, 500 cells from each transfection were seeded per well on 6 -well plates. They were incubated for additional 6 days at $37^{\circ} \mathrm{C}$ and stained with crystal violet (Acros Organics, Geel, Belgium, 229641000). Plates were scanned and the number of colonies was counted using Image $\mathbf{J}$ Software (National Institute of Health, Bethesda, MD, USA).

BrdU assay. Cells were transfected with control, Notch1 and/or p27 siRNA. After 48 h, 5,000 cells were seeded per well on 96-well plates. Following an additional 48 or $72 \mathrm{~h}$ incubation of the cells, they were labeled with BrdU using Cell Proliferation Biotrak ELISA System (GE Healthcare, RPN250) according to the manufacturer's instructions. The label was measured on Microplate Reader (Bio-Rad, 680) provided with Microplate Manager 5.2.1 software.

Pulse-chase experiment. For the pulse, the cells were washed with PBS and incubated for $16 \mathrm{~h}$ in methionine/cysteine-free $\mathrm{MEM}$, to which $500 \mu \mathrm{Ci}$ of [35S]-cysteine/methionine (Met-[35S]-label, Hartmann Analytic, Braunschweig, Germany) was added per plate. After labeling the cells were washed with PBS and chased with complete MEM for the indicated times. The radiolabeled cells were lysed in cold lysis buffer (30 mM Tris- $\mathrm{HCl}, 120 \mathrm{mM} \mathrm{NaCl}, 10 \%$ glycerol, $2 \mathrm{mM}$ EDTA, $2 \mathrm{mM} \mathrm{KCl}, 1 \%$ Triton X-100) containing protease inhibitors, ultrasonified and centrifuged (14,000 x g, $15 \mathrm{~min})$. Supernatants were adjusted to the same protein content with lysis buffer and pre-cleared with Sepharose ${ }^{\circledR}$ CL-4B beads
(Sigma, CL4B200). Samples were then incubated with $1.5 \mu \mathrm{g}$ of p27 antibody overnight at $4^{\circ} \mathrm{C}$. Immune complexes were precipitated with rec-Protein G-Sepharose ${ }^{\circledR}$ 4B Conjugate beads (Invitrogen, 101241) for $4 \mathrm{~h}$ at $4^{\circ} \mathrm{C}$. Precipitates were washed five times with cold lysis buffer. Proteins were released from the beads by incubation with $0.5 \mathrm{~N} \mathrm{NaOH}$ for $1 \mathrm{~h}$ at $37^{\circ} \mathrm{C}$. Finally protein samples were mixed with Ultima Gold ${ }^{\mathrm{TM}}$ scintillation cocktail (Perkin-Elmer, Rodgau, Germany) and counted for radioactivity in a liquid scintillation counter with automatic quench correction (Perkin-Elmer). Non-specific binding to the beads was determined by conducting the whole immunoprecipitation procedure without p27 antibody. All measurements were corrected for non-specific binding.

\section{Results}

Notch1 knockdown causes p27 stabilization in CRC cells. Notch1 receptor overexpression and aberrant pathway activation have previously been described in CRC (9). Additionally, it has been reported that the effects of Notch1 on cell fate involve the cell cycle regulator $\mathrm{p} 27(7,19)$. Therefore, we initially compared the levels of activated Notch1 (NICD1) and p27 in different CRC cell lines by immunoblot analysis (Fig. 1A). Amongst the seven analyzed cell lines, Caco 2 cells showed lowest NICD1 and highest p27 levels. Consistent with this reciprocal correlation between p27 and Notch1 expression, knockdown of Notch1 caused upregulation of p27 protein levels (Fig. 1B and C). Since the increased protein levels of p27 were not caused by significant alterations in p27 mRNA expression (Fig. 1D), we further characterized the putative post-transcriptional regulation of $\mathrm{p} 27$ by Notch1. Inhibition of protein translation using cycloheximide indicated significant stabilization of $\mathrm{p} 27$ protein over time when Notch1 was depleted (Fig. 1E). Consistently, pulse-chase analysis revealed more than 2.5-fold prolongation of the p27 half-life (from 7 to $18 \mathrm{~h}$ ) upon downregulation of Notch1 (Fig. 1F).

Notch1 regulates ubiquitin ligases responsible for p27 degradation. The most prominent mode of regulating p27 levels throughout the cell cycle is targeting p27 for proteasomal degradation. This is accomplished via ubiquitination by the Kip1 ubiquitination-promoting complex (KPC) in the cytoplasm in early G1 phase (15) and by the SKP1-CUL1-F-box protein $\left(\mathrm{SCF}^{\mathrm{SKP} 2}\right)$ in the nucleus in late $\mathrm{G} 1$ which allows the cell to proceed to $S$ phase (20). Taking these key mechanisms of p27 regulation into consideration, we analyzed the effects of Notch1 on the two subunits of the KPC-ubiquitin ligase, namely KPC1 and KPC2, and on S-phase kinase-associated protein 2 (SKP2), the F-box substrate-recognition subunit of $\mathrm{SCF}^{\mathrm{SKP} 2}$ complex. An immunoblot analysis showed that KPC1, KPC2 and SKP2 were downregulated after Notch1 depletion (Fig. 2A). This regulation occurs at least partially on the transcriptional level, since SKP2, KPC1 and KPC2 mRNA levels were diminished upon Notch1 downregulation (Fig. 2B). A subcellular fractionation revealed that in consequence of the regulation of both nuclear and cytoplasmic p27-targeting ubiquitin ligases, the levels of p27 increase in both cellular fractions (Fig. 2C). Similar to Notch1 knockdown, KPC1 and SKP2 knockdown resulted in an upregulation of p27 (Fig. 2D). Taken together, these results suggest that the regulation of p27 by Notch1 is mediated by KPC and/or SKP2. 
A

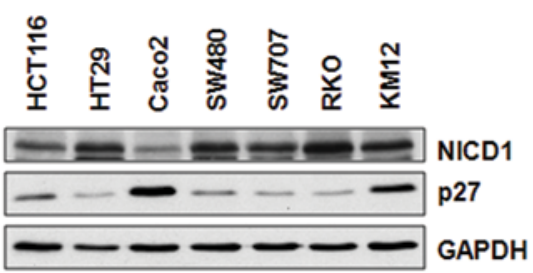

C

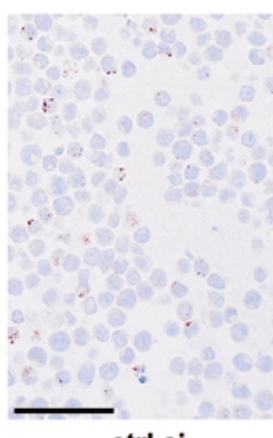

ctrl si

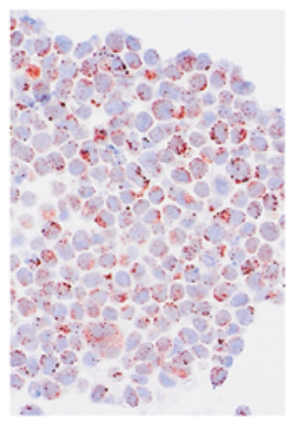

Notch1 si1
B

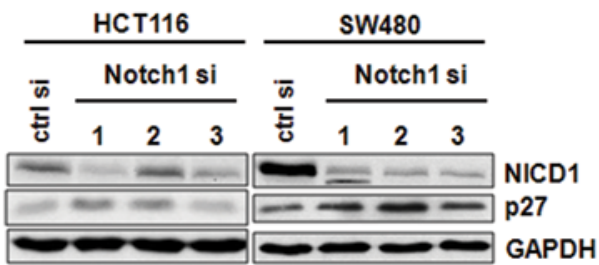

D
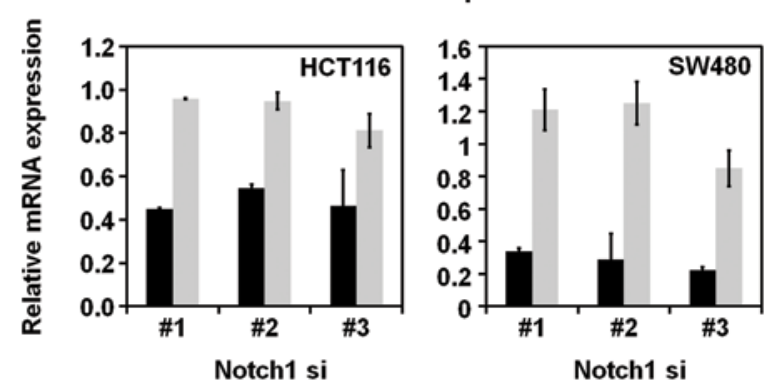

$\mathbf{E}$

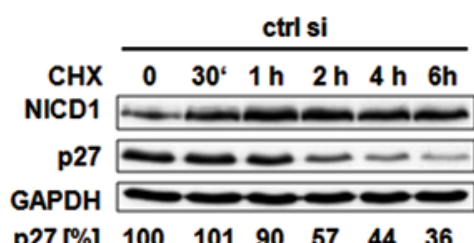

p27 [\%] $\begin{array}{llllll}100 & 101 & 90 & 57 & 44 & 36\end{array}$

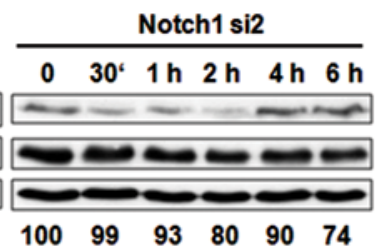

$\mathbf{F}$

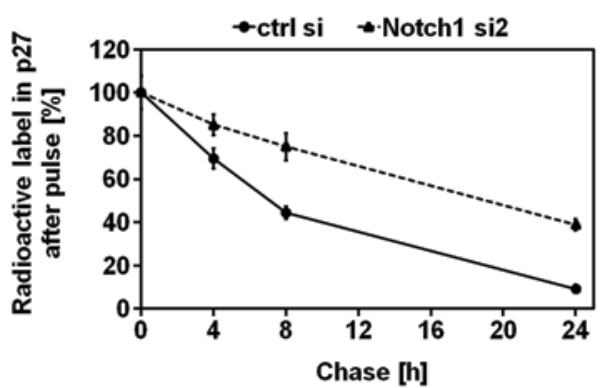

Figure 1. Notch1 knockdown causes p27 stabilization in CRC cells. (A) Immunoblot analysis of active Notch1 (NICD1) and p27 expression levels in a panel of CRC cell lines. Total soluble proteins $(50 \mu \mathrm{g})$ were separated per lane; GAPDH was used as a loading control. (B) Immunoblot analysis of p27 expression levels in control siRNA- and Notch1 siRNA-transfected HCT116 and SW480 cells. Cell lysates were prepared $72 \mathrm{~h}$ after siRNA transfection; $50 \mu \mathrm{g}$ total soluble proteins were separated per lane. (C) Immunohistochemical staining for $\mathrm{p} 27$ in SW480 cells $72 \mathrm{~h}$ after transfection with control siRNA or Notch1 siRNA1. Bar corresponds to $50 \mu \mathrm{m}$. (D) qRT-PCR analysis of Notch1 and p27 mRNA expression levels in HCT116 and SW480 cells. RNA was isolated $72 \mathrm{~h}$ after siRNA transfection. Expression data were normalized to internal 18S ribosomal RNA expression. Data are presented in relation to Notch1 and p27 expression in control siRNA-transfected cells $(\mathrm{n}=2$, mean $\pm \mathrm{SD})$. (E) Immunoblot analysis of control siRNA- and Notch1 siRNA-transfected Caco2 cells. Seventy-two hours post-transfection cells were treated with $\mathrm{CHX}(10 \mu \mathrm{g} / \mathrm{ml})$ for the indicated times. Total soluble proteins $(50 \mu \mathrm{g})$ were separated per lane. p27 expression levels were densitometrically quantified using Image J Software and were compared to those of lysates prepared at time 0 . (F) Pulse-chase analysis of Caco 2 cells $48 \mathrm{~h}$ post-transfection with control siRNA or Notch1 siRNA2. Cell lysates from the indicated time points were immunoprecipitated with p27 antibody and the incorporated radioactive [35S] methionine was quantified $(\mathrm{n}=3$, mean $\pm \mathrm{SD})$. For details, see Materials and methods.

p27 is a mediator of Notch1-dependent growth arrest. Since p27 is one of the key inhibitors of the cell cycle, we investigated the effects of its regulator, Notch1, on cellular growth. Furthermore, we analyzed to which extent p27 contributes to these effects. Notch1 knockdown inhibited cell proliferation as indicated by the decrease of BrdU-positive cells which was maintained over time (Fig. 3A). This was accompanied by a G2/M phase arrest (Fig. 3B). Finally, colony formation was inhibited by Notch1 downregulation (Fig. 3C). Importantly, all of these Notch1-dependent effects on cellular growth and proliferation could partially be compensated by knockdown of p27 (Fig. 3A-C). Further, to investigate the impact of p27 expression on proliferation in human tumor tissue, 44 CRC patients' samples were immunohistochemically analyzed. In accordance with the data obtained from cell culture experiments, samples expressing low levels of p27 were characterized by an increased proliferation capacity and vice versa (Fig. 3D). Interestingly, an upregulation of Notch1 and downregulation of p27 were observed at the infiltration zones of the carcinomas (Fig. 3E).

Notchl knockdown synergistically promotes cell death together with chemo- and radiotherapy. Various mechanisms for interference with Notch signaling have been investigated in CRC therapy (reviewed in ref. 8) and $\gamma$-secretase inhibitors (GSIs) have been tested in phase I and II clinical trials. Therefore, we were interested in analyzing the potential synergistic effects 
A

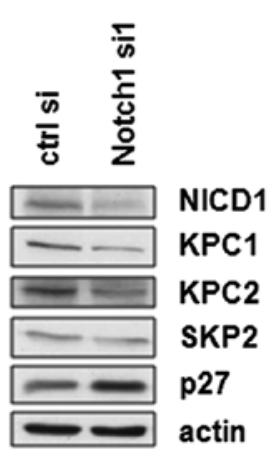

C
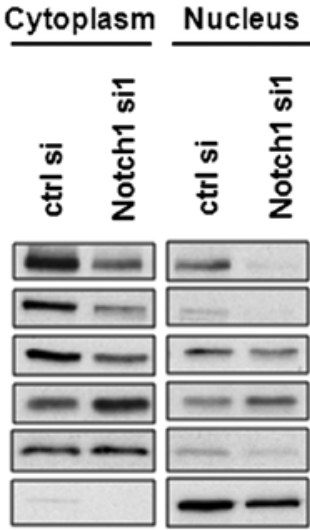

p27
B

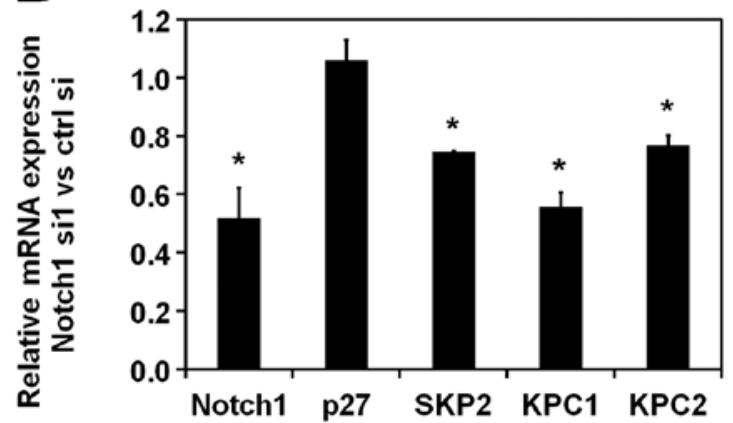

NICD1

D

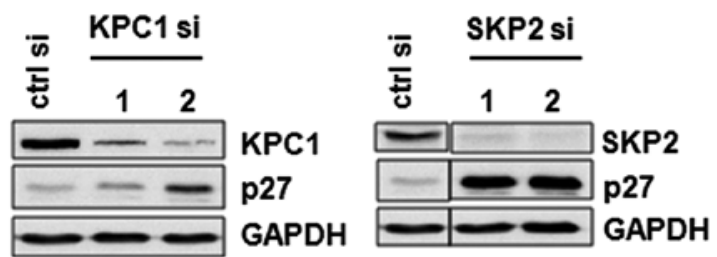

KPC1

SKP2

casp 3

PARP

Figure 2. Notch1 regulates ubiquitin ligases responsible for p27 degradation. (A) Immunoblot analysis of control siRNA- and Notch1 siRNA-transfected HCT116 cells. Cell lysates were prepared $72 \mathrm{~h}$ after siRNA transfection; $50 \mu \mathrm{g}$ total soluble proteins were separated per lane; $\beta$-actin was used as a loading control. (B) qRT-PCR analysis of mRNA expression levels in control siRNA- and Notch1 siRNA-transfected HCT116 cells. RNA was isolated 72 h after siRNA transfection. Expression data were normalized to internal 18S ribosomal RNA expression. Data are presented in relation to mRNA expression levels in control siRNA-treated cells $\left(\mathrm{n}=3\right.$, mean \pm SD, Student's t-test, $\left.{ }^{*} \mathrm{P}<0.05\right)$. (C) Immunoblot analysis of cytoplasmic and nuclear fractions of HCT116 cells $72 \mathrm{~h}$ post-transfection with control siRNA or Notch1 siRNA1. A total of $30 \mu \mathrm{g}$ cytoplasmic proteins and $10 \mu \mathrm{g}$ nuclear proteins were separated per lane; caspase 3 was used as cytoplasmic loading control and PARP as nuclear loading control. (D) Immunoblot analysis of SW480 cells transfected with a control siRNA, KPC1 siRNAs or SKP2 siRNAs, respectively. Lysates were prepared $72 \mathrm{~h}$ after transfection.

of Notch1 siRNA-mediated knockdown in combination with chemo- and radiotherapy. Compared to GSIs, the siRNA-mediated downregulation used in our study is specifically limited to the interference with Notch1 signaling. As shown in Fig. 4, siRNA-mediated suppression of Notch1 significantly increased cell death induced by 5 -fluorouracil (5-FU), oxaliplatin (OX) and ionizing radiation. Notch1 knockdown itself induced slight to moderate increase of cell death (control samples). In contrast, HCT116 cells showed only slight additive effects of Notch1 knockdown and conventional anticancer therapy (data not shown).

\section{Discussion}

Notch signaling plays a crucial role in cell proliferation but the exact underlying mechanisms are far from clear. Several studies have shown that in T-ALL and breast cancer Notch1 regulates cell cycle and progression via its direct target c-myc (21-24). Cyclin D1 was described to be a direct target of Notch1 and Jag1-mediated Notch signaling in breast cancer and RKE (rat kidney epithelial) cells (25-27), and cyclin D3 was shown to be an essential mediator of Notch tumorigenic role in T-ALL (28). In addition to cyclin D1, CDK2 was also demonstrated to be regulated by Notch in RKE cells (29). Accordingly, the CDKIs p21 and p57 are transcriptionally repressed by Notch signaling (7,30). Further, Notch signaling was described to regulate p27 transcription in CRC initiating cells which ensured their self-renewal (19).

In this study, we confirm that Notch1 suppresses the expression of p27 in colorectal carcinoma cells. Various mechanisms could account for this phenomenon. In fibroblasts and T-ALL cell lines Notch1 signaling transcriptionally activates the expression of ubiquitin ligase SKP2, thereby decreasing cellular p27 protein levels $(20,31)$. In intestinal crypt progenitor cells the transcriptional regulation of p27 by Notch1 signaling is mediated by Hes1 (7). In our study, the knockdown of Notch1 in the CRC cell lines did not cause a decrease in Hes1 mRNA probably due to the compensatory signaling by the other Notch receptors (data not shown). However, Notch1 knockdown induced a decrease in SKP2, KPC1 and KPC2 mRNA expression. KPC1 is the catalytic subunit of the KPC E3 ubiquitin-protein ligase complex, while $\mathrm{KPC} 2$ is the non- 

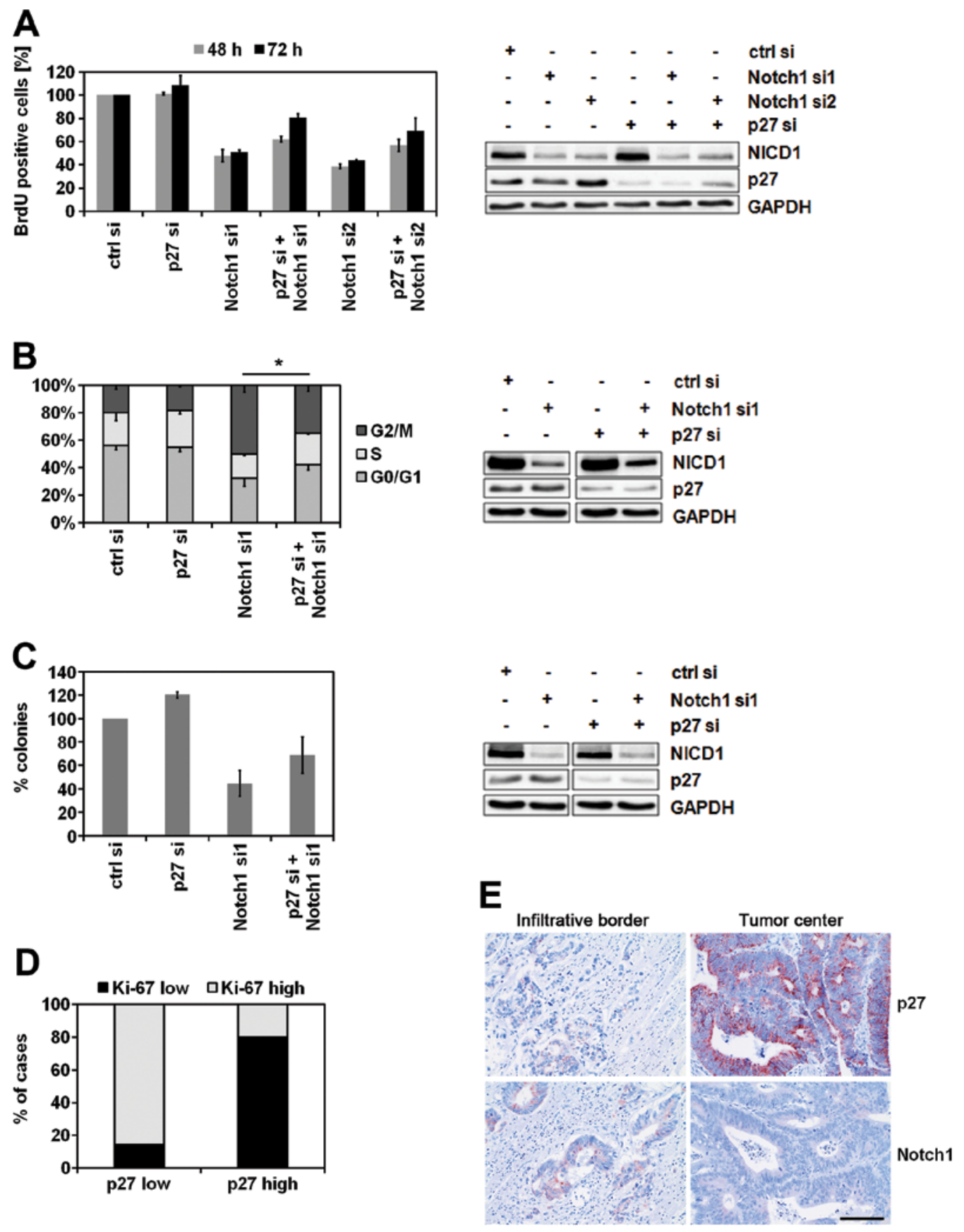

Figure 3. p27 is a mediator of Notch1-dependent growth arrest. (A) BrdU analysis of proliferating Caco2 cells. Forty-eight hours after siRNA transfection, Caco2 cells were seeded on 96-well plate and incubated for additional 48 or $72 \mathrm{~h}$. Then, the proliferating cells were labeled with BrdU and quantified spectrophotometrically $(\mathrm{n}=2$, mean $\pm \mathrm{SD}$ ) (left panel). Representative immunoblot analysis of Caco2 lysates prepared in parallel (right panel). Total soluble proteins ( $30 \mu \mathrm{g}$ ) were separated per lane. (B) Cell cycle analysis of SW480 cells. Seventy-two hours after siRNA-transfection the cells were stained with PI and their cell cycle distribution was analyzed by flow cytometry ( $\mathrm{n}=3$, mean $\pm \mathrm{SD}$, Student's $\mathrm{t}$-test, ${ }^{*} \mathrm{P}<0.05$ for all three cell cycle phases) (left panel). Representative immunoblot analysis of SW480 lysates prepared in parallel (right panel). Total soluble proteins ( $30 \mu \mathrm{g}$ ) were separated per lane. (C) Clonogenicity assay of HCT116 cells. Twenty-four hours after transfection with the indicated siRNAs, 500 cells from each transfection were seeded on a 6 -well plate and incubated for additional 6 days. Then the colonies were stained with crystal violet and counted using Image J Software after scanning of the plate ( $\mathrm{n}=2$, mean \pm SD) (left panel). Representative immunoblot analysis of HCT116 lysates prepared in parallel (right panel). Total soluble proteins $(30 \mu \mathrm{g})$ were separated per lane. (D) Association between p27 expression and proliferative activity in human CRC tissue samples. Tissue samples $(\mathrm{n}=44)$ were immunohistochemically stained with anti-p27 antibody and anti-Ki67 antibody. The cut-off value for low/high Ki67 was 50\% Ki67-positive tumor cells (group 'p27 low', n=14; group 'p27 high', n=30). (E) Immunohistochemical expression of Notch1 and p27 at the invasion front of CRC. In contrast to central parts of the tumor, at the infiltrative tumor border expression of p27 was typically low whereas Notch1 was strongly expressed by carcinoma cells (bar, $50 \mu \mathrm{m}$ ).

catalytic subunit of this complex. In contrast to SKP2, which is the substrate-recognition subunit of the SCF E3 ubiquitinprotein ligase complex, the KPC complex is localized in the cytoplasm. In accordance with our data KPC has been shown to accomplish p27 degradation in different cell types such as fibroblasts, cells of the nervous system and dendritic 
A

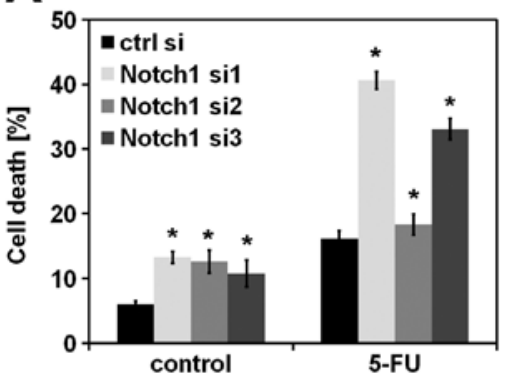

B

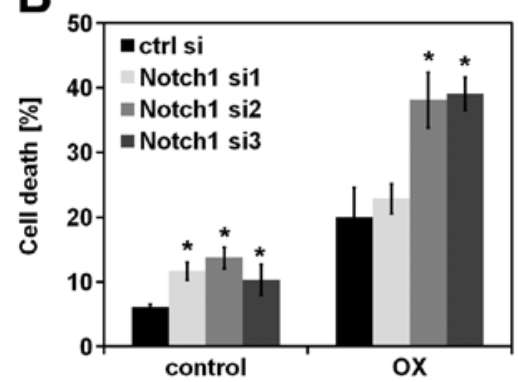

C

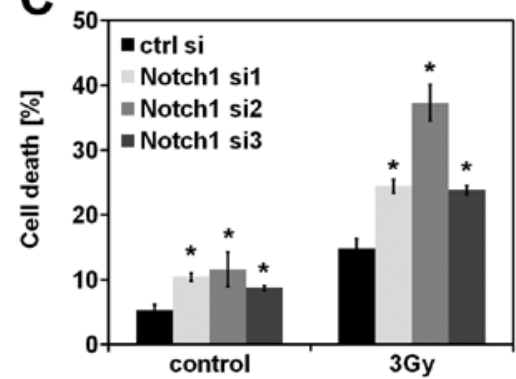

D

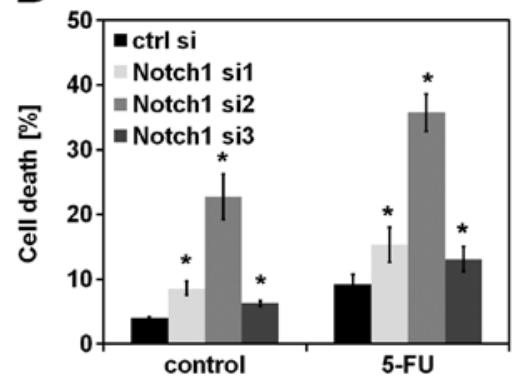

E

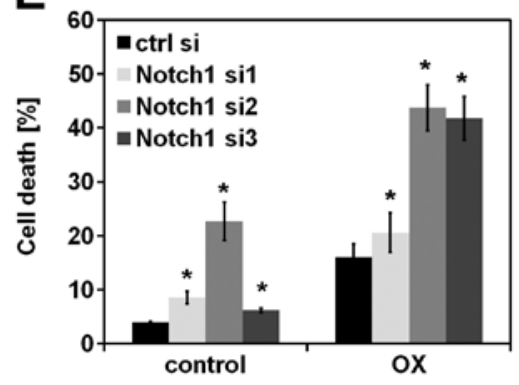

F

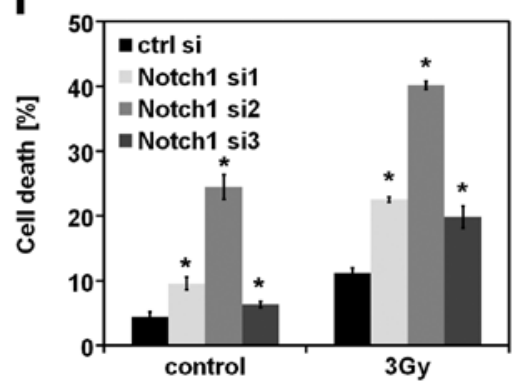

Figure 4. Knockdown of Notch1 synergistically increases cell death induced by chemo- and radiotherapy. (A, B and C) SW480 and (D, E and F) SW707 cells were transfected with the indicated siRNAs. Twenty-four hours later, the cells were treated with a chemotherapeutic drug, 5-FU (200 $\mu \mathrm{M})$ or oxaliplatin $(\mathrm{OX}, 100 \mu \mathrm{M})$, or irradiated ( $3 \mathrm{~Gy}$ ). After additional $48 \mathrm{~h}$ of incubation, cell death was quantified by flow cytometry analysis using PI staining ( $=3$, mean \pm SD, Student's t-test, $\left.{ }^{*} \mathrm{P}<0.01\right)$. P-values were calculated for each treatment comparing the Notch1 siRNA-transfected cells to the control siRNA-transfected cells.

cells (15,32-36). The KPC-mediated decrease of p27 in the cytoplasm is necessary for the cells to proceed from G0 to G1 phase, while the SCF-mediated downregulation of p27 in the nucleus is required for transition from $\mathrm{G} 1$ to $\mathrm{S}$ phase of the cell cycle. Notch1 regulation of both E3 ubiquitin-protein ligase complexes accounts for the altered levels of p27 in both the cytoplasmic and the nuclear cellular fractions. While previous reports have shown that the SKP2 promoter contains a NICD1-binding sequence, our study is the first one describing Notch1-dependent transcriptional regulation of $\mathrm{KPC1}$ and KPC2.

Further analyses revealed that the effects of Notch1 on cell cycle and cellular proliferation are at least partially mediated by $\mathrm{p} 27$. Notch1 knockdown induced $\mathrm{G} 2 / \mathrm{M}$ phase cell cycle arrest, decreased proliferation and diminished colony formation. However, the function of p27 might be supported by other cell cycle inhibitors from the CDKI family such as p21 and p57. In fact, we have observed a transcriptional regulation of $\mathrm{p} 21$ by Notch1 signaling leading to increase of p21 protein expression after Notch1 knockdown (data not shown). Furthermore, tran- scriptional regulation of p57 by the Notch target gene Hes1 has previously been described in intestinal crypt progenitor cells (7). Hes1 was also shown to bind p57 promoter and repress p57 transcription, thereby avoiding senescence in hepatocellular carcinoma (37).

Although the major role of p27 is to inhibit the cell cycle, this is not its only function in the cell. Interestingly, cytoplasmic localization of $\mathrm{p} 27$ has been associated with enhanced cell motility because of reduced activity of the small GTPase RhoA (38). Therefore the subcellular localization is a crucial criterion whether p27 will play the role of a tumor suppressor or an oncogene. Highly aggressive tumors are often characterized by reduced nuclear p27 which enhances proliferation and by mislocalized cytoplasmic p27 which drives invasion (39). Several studies in CRC found that reduced p27 protein levels are associated with poor outcome, including disease recurrence or death (40-43). Others have reported that cytoplasmic p27 correlated with high nuclear p27 staining and good prognosis in CRC patients (44). In addition to being used as prognostic marker, it has been proposed that $\mathrm{p} 27$ has predic- 
tive potential for response to cancer therapy. Increase of nuclear p27 levels or shift of p27 from the cytoplasm to the nucleus might be a predictor for response to inhibitors of p27 upstream regulators such as MEK/Src or PI3K/mTOR respectively $(45,46)$.

In addition to the inhibitory effects of Notch1 knockdown on proliferation and cell cycle regulation, our study provides evidence, that high levels of Notch1 render CRC cells resistant to cell death induced by cytotoxic drugs and ionizing radiation. Notch signaling has previously been described to regulate antiapoptotic proteins such as Mcl-1, Bcl-2, Bcl- $\mathrm{x}_{\mathrm{L}}$ and XIAP $(47,48)$, thereby inhibiting apoptosis. Additionally, Notch-dependent inhibition of apoptosis can also take place through negative regulation of p53 and PTEN $(23,49)$. Inhibition of Notch signaling has emerged as a promising future therapeutic approach. GSIs are currently used in phase I and II clinical trials for CRC patients (RO4929097, Roche, NCT01198535, NCT01158274, NCT01131234; NCT01270438, NCT01116687) and other malignancies such as breast cancer, pancreatic cancer, T-ALL, and brain tumors (MK0752, Merck, NCT00645333, NCT01098344, NCT00100152, NCT00572182). There is growing evidence that the combination of Notch signaling inhibition and chemotherapy, radiotherapy or inhibition of other pathways being crucial for tumor maintenance will synergize together in order to kill CRC cells $(50,51)$. This is in fact the rationale of many ongoing clinical trials. For example, the combination of cetuximab (a monoclonal antibody blocking the EGFR) and the GSI RO4929097 is used in a phase I clinical trial in patients with metastatic CRC (NCT01198535). RO4929097 in combination with chemotherapy (oxaliplatin, leucovorin calcium, 5-FU) and bevacizumab (a monoclonal antibody inhibiting VEGF-A) is used in a phase II clinical trial in patients with the same malignancy (NCT01270438). Since Notch signaling plays a crucial role in CRC initiating cells (so-called colorectal cancer stem cells), it further represents an attractive therapeutic target especially with regard to the highly resistant cancer initiating cells (52).

Unfortunately, GSIs target all Notch receptors as well as the other substrates of the $\gamma$-secretase. Thus, significant side-effects of GSIs, such as gastro-intestinal toxicity and diarrhea, were observed in clinical trials (53) due to the inhibition of Notch signaling causing the differentiation of the intestinal progenitor cells into post-mitotic secretory goblet cells (54). Therefore, other methods for more specific inhibition of individual players within the Notch signaling pathway are explored. For instance, a monoclonal antibody against the Delta-like ligand 4 (DLL4), called MEDI0639, is used in a phase I clinical trial in advanced solid tumors (NCT01577745). The expected antitumor effect of this antibody is due to inhibition of tumor angiogenesis (3). Notch1 and Notch2 specific monoclonal antibodies (anti-NRR1 and anti-NRR2) stabilizing the receptor negative regulatory region (ADAM/S2-cleavage region) are holding promise for successful specific inhibition of Notch signaling, thereby avoiding the severe intestinal toxicity of the dual receptor inhibition (55).

In conclusion, our study provides initial evidence that specific interference with Notch1 signaling could be sufficient to sensitize $\mathrm{CRC}$ cells to 5-FU, oxaliplatin and ionizing radiation, which are routinely used in the clinical practice.
Downregulation of Notch1 receptor leads to increased p27 levels and analyzing the subcellular localization of this cell cycle inhibitor might be important for predicting treatment success. Further investigation on the mechanism of regulation of KPC1 and KPC 2 by Notch 1 might also be an attractive topic. Collectively, we describe possible mechanisms of regulation of CRC cell growth by Notch1 signaling and investigating these complex interactions is crucial for using Notch1 inhibition for specifically targeting the death of cancer cells with mild side-effects for the patients.

\section{Acknowledgements}

We thank Sarah Messnard and Heike Conrad for their technical assistance. We further thank Stephan Macher-Goeppinger for his expert advice on immunohistochemistry.

\section{References}

1. Bray SJ: Notch signalling: a simple pathway becomes complex. Nat Rev Mol Cell Biol 7: 678-689, 2006.

2. Radtke F and Raj K: The role of Notch in tumorigenesis: oncogene or tumour suppressor? Nat Rev Cancer 3: 756-767, 2003.

3. Benedito R, Roca C, Sorensen I, et al: The notch ligands Dll4 and Jagged1 have opposing effects on angiogenesis. Cell 137: 1124-1135, 2009.

4. Fre S, Huyghe M, Mourikis P, Robine S, Louvard D and Artavanis-Tsakonas S: Notch signals control the fate of immature progenitor cells in the intestine. Nature 435: 964-968, 2005.

5. Schroder N and Gossler A: Expression of Notch pathway components in fetal and adult mouse small intestine. Gene Expr Patterns 2: 247-250, 2002.

6. Jensen J, Pedersen EE, Galante P, et al: Control of endodermal endocrine development by Hes-1. Nat Genet 24: 36-44, 2000.

7. Riccio O, van Gijn ME, Bezdek AC, et al: Loss of intestinal crypt progenitor cells owing to inactivation of both Notch1 and Notch 2 is accompanied by derepression of CDK inhibitors p27Kip1 and p57Kip2. EMBO Rep 9: 377-383, 2008.

8. Qiao L and Wong BC: Role of Notch signaling in colorectal cancer. Carcinogenesis 30: 1979-1986, 2009.

9. Zhang Y, Li B, Ji ZZ and Zheng PS: Notch1 regulates the growth of human colon cancers. Cancer 116: 5207-5218, 2010.

10. Zolkiewska A: ADAM proteases: ligand processing and modulation of the Notch pathway. Cell Mol Life Sci 65: 2056-2068, 2008.

11. Fortini ME: Gamma-secretase-mediated proteolysis in cellsurface-receptor signalling. Nat Rev Mol Cell Biol 3: 673-684, 2002.

12. Katoh $M$ and Katoh $M$ : Integrative genomic analyses on HES/HEY family: Notch-independent HES1, HES3 transcription in undifferentiated ES cells, and Notch-dependent HES1, HES5, HEY1, HEY2, HEYL transcription in fetal tissues, adult tissues, or cancer. Int J Oncol 31: 461-466, 2007.

13. Chu IM, Hengst L and Slingerland JM: The Cdk inhibitor p27 in human cancer: prognostic potential and relevance to anticancer therapy. Nat Rev Cancer 8: 253-267, 2008.

14. Lu Z and Hunter T: Ubiquitylation and proteasomal degradation of the p21(Cip1), p27(Kip1) and p57(Kip2) CDK inhibitors. Cell Cycle 9: 2342-2352, 2010.

15. Kamura T, Hara T, Matsumoto M, et al: Cytoplasmic ubiquitin ligase KPC regulates proteolysis of p27(Kip1) at G1 phase. Nat Cell Biol 6: 1229-1235, 2004

16. Ishida N, Hara T, Kamura T, Yoshida M, Nakayama K and Nakayama KI: Phosphorylation of p27Kip1 on serine 10 is required for its binding to CRM1 and nuclear export. J Biol Chem 277: 14355-14358, 2002.

17. Castro F, Dirks WG, Fahnrich S, Hotz-Wagenblatt A, Pawlita M and Schmitt M: High-throughput SNP-based authentication of human cell lines. Int J Cancer 132: 308-314, 2013.

18. Schmitt M and Pawlita M: High-throughput detection and multiplex identification of cell contaminations. Nucleic Acids Res 37: e119, 2009. 
19. Sikandar SS, Pate KT, Anderson S, et al: NOTCH signaling is required for formation and self-renewal of tumor-initiating cells and for repression of secretory cell differentiation in colon cancer. Cancer Res 70: 1469-1478, 2010.

20. Sarmento LM, Huang H, Limon A, et al: Notch1 modulates timing of G1-S progression by inducing SKP2 transcription and p27 Kip1 degradation. J Exp Med 202: 157-168, 2005.

21. Sharma VM, Calvo JA, Draheim KM, et al: Notch1 contributes to mouse T-cell leukemia by directly inducing the expression of c-myc. Mol Cell Biol 26: 8022-8031, 2006.

22. Weng AP, Millholland JM, Yashiro-Ohtani Y, et al: c-Myc is an important direct target of Notch1 in T-cell acute lymphoblastic leukemia/lymphoma. Genes Dev 20: 2096-2109, 2006.

23. Palomero T, Sulis ML, Cortina M, et al: Mutational loss of PTEN induces resistance to NOTCH1 inhibition in T-cell leukemia. Nat Med 13: 1203-1210, 2007.

24. Klinakis A, Szabolcs M, Politi K, Kiaris H, ArtavanisTsakonas S and Efstratiadis A: Myc is a Notch1 transcriptional target and a requisite for Notch1-induced mammary tumorigenesis in mice. Proc Natl Acad Sci USA 103: 9262-9267, 2006.

25. Ling H, Sylvestre JR and Jolicoeur P: Notch1-induced mammary tumor development is cyclin D1-dependent and correlates with expansion of pre-malignant multipotent duct-limited progenitors. Oncogene 29: 4543-4554, 2010.

26. Cohen B, Shimizu M, Izrailit J, et al: Cyclin D1 is a direct target of JAG1-mediated Notch signaling in breast cancer. Breast Cancer Res Treat 123: 113-124, 2010.

27. Stahl M, Ge C, Shi S, Pestell RG and Stanley P: Notchl-induced transformation of RKE-1 cells requires up-regulation of cyclin D1. Cancer Res 66: 7562-7570, 2006.

28. Sicinska E, Aifantis I, Le Cam L, et al: Requirement for cyclin D3 in lymphocyte development and T cell leukemias. Cancer Cell 4: 451-461, 2003.

29. Ronchini $C$ and Capobianco AJ: Induction of cyclin D1 transcription and CDK2 activity by Notch(ic): implication for cell cycle disruption in transformation by Notch(ic). Mol Cell Biol 21: 5925-5934, 2001.

30. Gotte M, Greve B, Kelsch R, et al: The adult stem cell marker Musashi-1 modulates endometrial carcinoma cell cycle progression and apoptosis via Notch-1 and p21WAF1/CIP1. Int J Cancer 129: 2042-2049, 2011.

31. Dohda T, Maljukova A, Liu L, et al: Notch signaling induces SKP2 expression and promotes reduction of p27Kip1 in T-cell acute lymphoblastic leukemia cell lines. Exp Cell Res 313: 3141-3152, 2007

32. Hara T, Kamura T, Kotoshiba S, et al: Role of the UBL-UBA protein KPC2 in degradation of p27 at G1 phase of the cell cycle. Mol Cell Biol 25: 9292-9303, 2005.

33. Lu Y, Adegoke OA, Nepveu A, et al: USP19 deubiquitinating enzyme supports cell proliferation by stabilizing KPC1, a ubiquitin ligase for p27Kip1. Mol Cell Biol 29: 547-558, 2009.

34. Zhao J, Zhang S, Wu X, et al: KPC1 expression and essential role after acute spinal cord injury in adult rat. Neurochem Res 36: 549-558, 2011

35. Lu C, Huang X, Zhang $\mathrm{X}$, et al: $\mathrm{miR}-221$ and miR-155 regulate human dendritic cell development, apoptosis, and IL-12 production through targeting of p27kip1, KPC1, and SOCS-1. Blood 117: 4293-4303, 2011 .

36. Susaki E, Nakayama K and Nakayama KI: Cyclin D2 translocates p27 out of the nucleus and promotes its degradation at the G0-G1 transition. Mol Cell Biol 27: 4626-4640, 2007.

37. Giovannini C, Gramantieri L, Minguzzi M, et al: CDKN1C/P57 is regulated by the Notch target gene Hes1 and induces senescence in human hepatocellular carcinoma. Am J Pathol 181: 413-422, 2012.
38. See WL, Heinberg AR, Holland EC and Resh MD: p27 deficiency is associated with migration defects in PDGF-expressing gliomas in vivo. Cell Cycle 9: 1562-1567, 2010.

39. Wander SA, Zhao D and Slingerland JM: p27: a barometer of signaling deregulation and potential predictor of response to targeted therapies. Clin Cancer Res 17: 12-18, 2011.

40. Noguchi T, Kikuchi R, Ono K, Takeno S, Moriyama H and Uchida Y: Prognostic significance of p27/kipl and apoptosis in patients with colorectal carcinoma. Oncol Rep 10: 827-831, 2003.

41. Manne U, Jhala NC, Jones J, et al: Prognostic significance of p27(kip-1) expression in colorectal adenocarcinomas is associated with tumor stage. Clin Cancer Res 10: 1743-1752, 2004.

42. Wu JT, Kakar S, Nelson RL, et al: Prognostic significance of DCC and p27Kip1 in colorectal cancer. Appl Immunohistochem Mol Morphol 13: 45-54, 2005.

43. Rosati G, Chiacchio R, Reggiardo G, De Sanctis D and Manzione L: Thymidylate synthase expression, p53, bcl-2, Ki-67 and p27 in colorectal cancer: relationships with tumor recurrence and survival. Tumour Biol 25: 258-263, 2004.

44. Watson NF, Durrant LG, Scholefield JH, et al: Cytoplasmic expression of p27(kip1) is associated with a favourable prognosis in colorectal cancer patients. World J Gastroenterol 12: 6299-6304, 2006

45. Gysin S, Lee SH, Dean NM and McMahon M: Pharmacologic inhibition of $\mathrm{RAF} \rightarrow \mathrm{MEK} \rightarrow \mathrm{ERK}$ signaling elicits pancreatic cancer cell cycle arrest through induced expression of p27Kip1. Cancer Res 65: 4870-4880, 2005.

46. Hong F, Larrea MD, Doughty C, Kwiatkowski DJ, Squillace R and Slingerland JM: mTOR-raptor binds and activates SGK1 to regulate p27 phosphorylation. Mol Cell 30: 701-711, 2008.

47. Fassl A, Tagscherer KE, Richter J, et al: Notch1 signaling promotes survival of glioblastoma cells via EGFR-mediated induction of anti-apoptotic Mcl-1. Oncogene 31: 4698-4708, 2012.

48. Liu WH, Hsiao HW, Tsou WI and Lai MZ: Notch inhibits apoptosis by direct interference with XIAP ubiquitination and degradation. EMBO J 26: 1660-1669, 2007.

49. Beverly LJ, Felsher DW and Capobianco AJ: Suppression of p53 by Notch in lymphomagenesis: implications for initiation and regression. Cancer Res 65: 7159-7168, 2005.

50. Meng RD, Shelton CC, Li YM, et al: gamma-Secretase inhibitors abrogate oxaliplatin-induced activation of the Notch-1 signaling pathway in colon cancer cells resulting in enhanced chemosensitivity. Cancer Res 69: 573-582, 2009.

51. Akiyoshi T, Nakamura M, Yanai K, et al: Gamma-secretase inhibitors enhance taxane-induced mitotic arrest and apoptosis in colon cancer cells. Gastroenterology 134: 131-144, 2008.

52. Pannuti A, Foreman K, Rizzo P, et al: Targeting Notch to target cancer stem cells. Clin Cancer Res 16: 3141-3152, 2010.

53. Real PJ, Tosello V, Palomero T, et al: Gamma-secretase inhibitors reverse glucocorticoid resistance in $\mathrm{T}$ cell acute lymphoblastic leukemia. Nat Med 15: 50-58, 2009.

54. Van Es JH, van Gijn ME, Riccio O, et al: Notch/gammasecretase inhibition turns proliferative cells in intestinal crypts and adenomas into goblet cells. Nature 435: 959-963, 2005.

55. Wu Y, Cain-Hom C, Choy L, et al: Therapeutic antibody targeting of individual Notch receptors. Nature 464: 1052-1057, 2010. 